\title{
Vitiligo penis isolated or penile vitiligo
}

Jihane Ziani, Sara Dahouki, Sara Elloudi, Hanane Baybay, Fatima Zehra Mernissi

Department of Dermatology, University Hospital Hassan II of Fez Morocco.

*Corresponding Author: Jihane Ziani, Department of Dermatology, University Hospital Hassan II of Fez Morocco.

Received date: March 11, 2020; Accepted date: March 23, 2020; Published date: March 27,2020

Citation: Jihane Z, Sara D, Sara E, Hanane B, Fatima Z Mernissi (2020).Vitiligo penis isolated or penile vitiligo.Journal of Dermatology and Dermatitis.5(1); Doi: 10.31579/2578-8949/65

Copyright: (92020 Jihane Ziani, This is an open-access article distributed under the terms of The Creative Commons. Attribution License, which permits unrestricted use, distribution, and reproduction in any medium, provided the original author and source are credited.
Abstract
Context: vitiligo with a unique location in the penis is a rare entity.
Presentation of the case: A 34-year-old man who presented himself in our training, he complained of a whitish plaque on his penis. The physical examination showed an achromic macule on the level of the penis, with a catch of contrast in light of Wood.
A skin biopsy of the dorsal surface of the penis showed a total absence of melanocytes and melanin granules in the basal layer.
Conclusion: This is a rare report of a case of vitiligo diagnosed by examination in light of Wood, with an isolated location in the penis.
Keywords: vitiligo, penis, wood light.

It is a 34-year-old man who presented himself in our training, for possible management of a whitish spot on his penis evolving for 12 years. The patient reports the appearance of the lesion concomitantly with sexual intercourse after his marriage, without family history of a similar case. Physical examination showed an achromic macula well limited to regular contours sitting on the dorsal side of the penis (Figure 1),

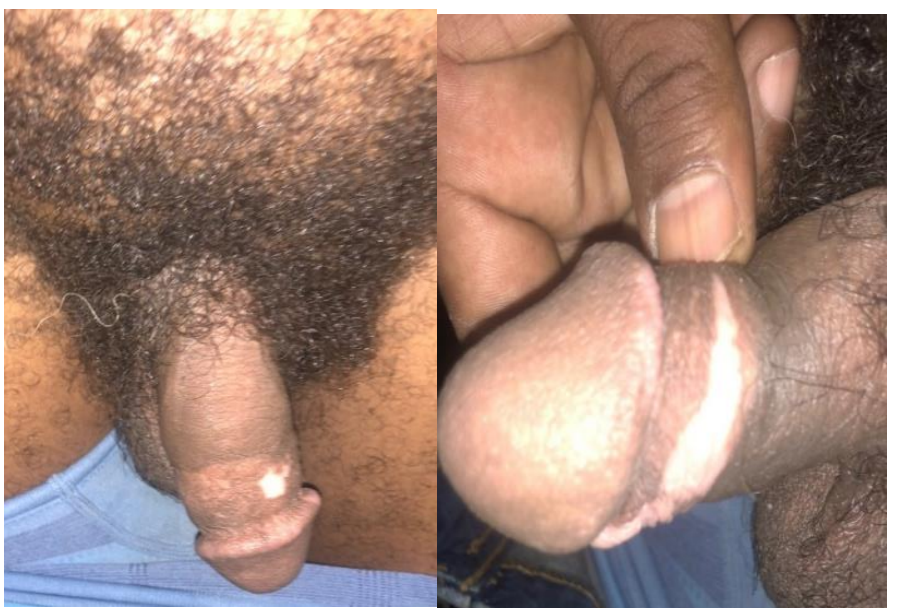

Figure 1 : Achromic macule on the level of the penis.

with a contrast enhancement when analyzed by Wood's light (Figure 2).

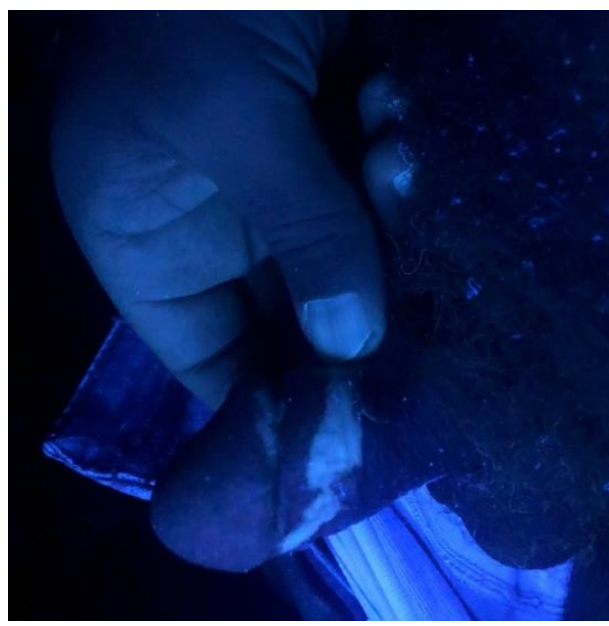

Figure 2 : Wood's light accentuated the depigmented area.

The rest of the skin and mucosal examination did not show similar lesions.

A skin biopsy of the dorsal surface of the penis showed a total absence of melanocytes and melanin granules in the basal layer. The dermis was normal.

Vitiligo is a skin condition characterized by depigmentation of the skin and mucous membranes, producing well-defined and depigmented macules and hypochromics, often localized in the areas of friction and Kobner. The etiology of vitiligo is unknown, but research suggests that it may involve autoimmune, genetic and other factors, explaining its 
frequent association with autoimmune diseases and disorders such as thyroid abnormalities, pernicious anemia, lupus disseminated erythematosus and other diseases. (1)

Vitiligo can affect skin in any part of the body such as hands, arms, face, genital areas including the penis in men and labia in women, which are a Kobner areas. If it affects the penis, it is called penile vitiligo. Vitiligo of the penis usually appears as penile discoloration on the foreskin, and sometimes though less commonly penile discoloration on the glans (head) of the penis. Vitiligo is treated with medications, topical creams, laser therapies and surgical methods. However, symptoms of vitiligo may reappear after treatment and new spots or plaques may appear in the same place or in a different place. Corticosteroid creams can reduce the amount of skin lightening, but they can lighten the skin with regular use and cause other penile skin problems. Unlike topical therapy with Tacrolimus which can be applied without side effects on the genital mucosa.

The particularity of our observation is the unique presentation of vitiligin disease on the penis in our case.

Conflicts of interest: Author declares that there is no conflict of interest

\section{References:}

1. Li, W., Xin, H., Ge, L., Song, H., \& Cao, W. (2014). Induction of vitiligo after imiquimod treatment of condylomata acuminata. BMC Infectious Diseases, 14(1). doi:10.1186/1471-2334-14-329 